Check for updates

Cite this: DOI: 10.1039/c9cc01937e

Received 9th March 2019,

Accepted 7th May 2019

DOI: $10.1039 / c 9 c c 01937 e$

rsc.li/chemcomm

\section{Bismuth oxychloride nanoflake assemblies as a new anode for potassium ion batteries $\uparrow$}

\author{
Wei Li, ${ }^{a}$ Yang $X u$, (D) ${ }^{\text {bc }}$ Yulian Dong, ${ }^{a}$ Yuhan Wu, (D) ${ }^{\mathrm{b}}$ Chenglin Zhang, ${ }^{\mathrm{b}}$ Min Zhou, \\ Qun Fu, ${ }^{a}$ Minghong $\mathrm{Wu}^{* a}$ and Yong Lei (D) ${ }^{\mathrm{b}}$
}

This work reports the first demonstration of bismuth oxyhalides as anode materials in potassium-ion batteries. BiOCl nanoflake assemblies deliver high capacities of $367 \mathrm{~mA} \mathrm{~h} \mathrm{~g}^{-1}$ at $50 \mathrm{~mA} \mathrm{~g}^{-1}$ and $175 \mathrm{~mA} \mathrm{~h} \mathrm{~g}^{-1}$ at $1 \mathrm{~A} \mathrm{~g}^{-1}$. The formation of $\mathrm{K}-\mathrm{Bi}$ alloys at an early stage of potassiation is obsenved.

Investigation of the feasibility of K-ion batteries (KIBs) has been greatly prompted during the past few years. ${ }^{1-5}$ There are two important advantages of KIBs that encourage researchers to move from Li-ion batteries (LIBs) to KIBs. First, $\mathrm{K}$ has a lower standard redox potential than $\mathrm{Li}$ in nonaqueous electrolytes, which can be translated to a higher cell voltage of KIBs. ${ }^{1}$ Second, nonaqueous $\mathrm{K}$ electrolytes have a higher ionic conductivity than Li electrolytes and lower interfacial reaction resistance derived from the lower desolvation activation energy. ${ }^{2,3} \mathrm{~A}$ study of KIB cathodes is mainly focused on intercalation-type materials, ${ }^{6,7}$ but there is a great variety of KIB anodes because of the various electrochemical mechanisms to store K-ions. ${ }^{1,4,8}$ It can be anticipated that the mechanisms in KIBs might be beyond our expectations and different from those in LIBs even when given the same material. Despite the diverse mechanisms, two criteria are important to obtain satisfactory KIB performance: (i) suitable ion diffusion pathways exist in the anode's structure to ensure fast ionic transport and (ii) high electronic conductivity is achievable to ensure fast electronic transport.

As a group of V-VI-VII ternary compounds, bismuth oxyhalides $\operatorname{BiOX}(\mathrm{X}=\mathrm{Cl}, \mathrm{Br}$ and $\mathrm{I})$ are a classic type of layer-structured materials. They crystallize into a tetragonal matlockite (PbFCl-type)

\footnotetext{
${ }^{a}$ Institute of Nanochemistry and Nanobiology, School of Environmental and Chemical Engineering, Shanghai University, Shanghai 200444, China.

E-mail:mhwu@shu.edu.cn

${ }^{b}$ Fachgebiet Angewandte Nanophysik, Institut für Physik \& ZMN MacroNano (ZIK),

Technische Universität Ilmenau, Ilmenau 98693, Germany.

E-mail: yong.lei@tu-ilmenau.de

${ }^{c}$ Department of Chemistry, University College London, London WC1H OAJ, UK.

E-mail:y.xu.1@ucl.ac.uk

$\dagger$ Electronic supplementary information (ESI) available: Experimental details, TEM, SEM after ultrasonic treatment and cycling, survey XPS, O 1s and Cl 2p XPS after the first cycle, and schematic of reaction pathways. See DOI: 10.1039/c9cc01937e
}

structure (space group P4/nmm) that consists of [X-Bi-O-Bi-O-X] slices stacked by the van der Waals interaction through the halogen atoms along the $c$ axis. ${ }^{9,10}$ The layered structure features strong intralayer covalent bonding and weak interlayer van der Waals interaction, which results in highly anisotropic electrical, mechanical and optical properties, endowing BiOX with potential applications in various fields. ${ }^{11-15}$ However, there have been very few studies on BiOX in the application of rechargeable alkalimetal ion batteries $\left(\right.$ LIBs $^{16,17}$ and Na-ion batteries (NIBs) $\left.{ }^{18}\right)$. It is surprising because BiOX meets the two criteria. On the one hand, the van der Waals gaps in BiOX naturally serve as twodimensional (2D) ion diffusion pathways. On the other hand, metallic Bi can be formed by the conversion reaction to enhance the electronic conductivity of the electrode matrix. Thus, it is urgent yet rational to exploit the potential of BiOX in the emerging field of KIBs.

In this work, we report the first application of BiOX compounds in KIBs by taking BiOCl as a demonstrator. BiOCl nanoflake assemblies (NFAs) are prepared and characterized by a submicron hierarchical structure that consists of square-shaped flake nanounits. The electrochemical mechanism study reveals the formation of K-Bi alloys at the initial stage of potassiation, which has not been observed in lithiation and sodiation of BiOX. BiOCl NFAs deliver a high capacity of $367 \mathrm{~mA} \mathrm{~h} \mathrm{~g}^{-1}$ and retain $58 \%$ capacity after 50 cycles at $50 \mathrm{~mA} \mathrm{~g}^{-1}$. They also show great rate capability by delivering a capacity of $175 \mathrm{~mA} \mathrm{~h} \mathrm{~g}{ }^{-1}$ at $1 \mathrm{~A} \mathrm{~g}^{-1}$. To the best of our knowledge, the NFAs exhibit the best electrochemical performance among all the BiOX compounds tested in alkali-metal ion batteries.

BiOCl NFAs are fabricated by a solvothermal reaction, where $\mathrm{NH}_{4} \mathrm{Cl}$ not only serves as the $\mathrm{Cl}$ precursor, but also lowers the $\mathrm{pH}$ value of the reaction solution to reduce the hydrolysis rate of $\mathrm{Bi}\left(\mathrm{NO}_{3}\right)_{3} \cdot 5 \mathrm{H}_{2} \mathrm{O} .{ }^{19}$ The lowered hydrolysis rate and the viscosity of ethylene glycol (EG) as the reaction solvent can affect the growth of the nanoflakes and lead to the formation of assemblies. ${ }^{20}$ The X-ray diffraction (XRD) pattern (Fig. 1a) can be well indexed to tetragonal phase BiOCl (JCPDS No. 06-0249) with a space group of $P 4 / \mathrm{nmm}$. Good crystallinity can be verified by the sharpness of the peaks. ${ }^{21,22}$ (001), (002) and (003) peaks 

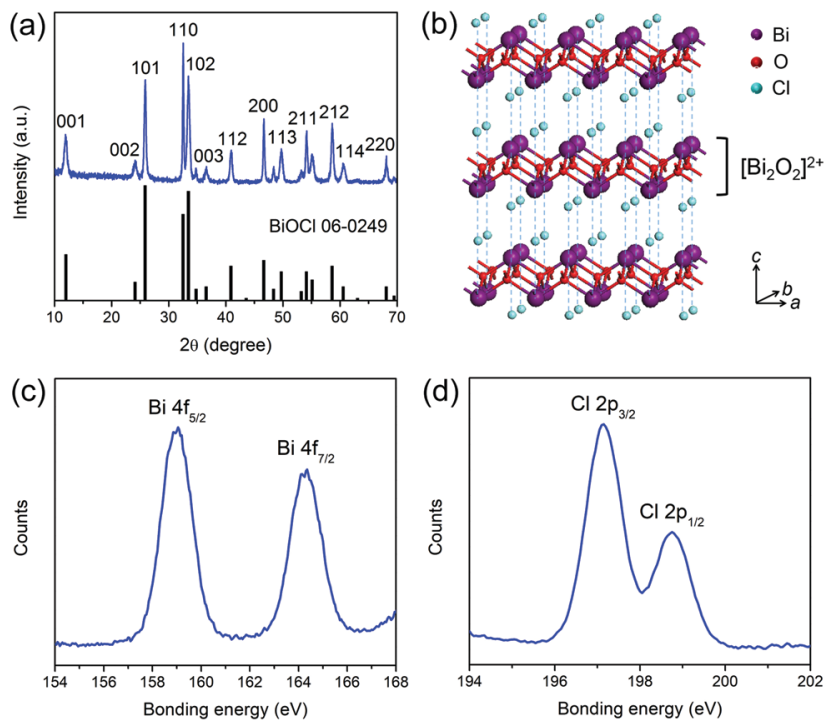

Fig. 1 (a) XRD pattern, (b) crystal structure, (c) Bi 4f, and (d) $\mathrm{Cl} 2 \mathrm{p}$ XPS spectra of BiOCI NFAs.

are broader than (110) and (200) peaks, indicating that the crystallite size along the $c$-axis, which is regarded as the thickness of the flakes, is smaller than that of the $a$ and $b$ plane, as will be seen in Fig. 2. The relatively strong (110) peak suggests that the flakes favour the growth along the (110) orientations which are perpendicular to the $c$-axis, forming thin slabs. ${ }^{23} \mathrm{BiOCl}$ has a typical layered structure (Fig. 1b) where $\left[\mathrm{Bi}_{2} \mathrm{O}_{2}\right]^{2+}$ slabs stack along the $c$ axis while being sandwiched by two slabs of $\mathrm{Cl}^{-}$, forming $[\mathrm{Cl}-\mathrm{Bi}-\mathrm{O}-\mathrm{Bi}-\mathrm{Cl}]$ units along the same axis. This enables the exposure of (001) facets and the $2 \mathrm{D}$ K-ion diffusion pathways between the $\left[\mathrm{Bi}_{2} \mathrm{O}_{2}\right]^{2+}$ slabs. The X-ray photoelectron spectroscopy (XPS) survey spectrum (Fig. S1, ESI $†$ ) shows the presence of only $\mathrm{Bi}, \mathrm{O}, \mathrm{Cl}$ and $\mathrm{C}$. The Bi $4 \mathrm{f}$ spectrum (Fig. 1c) shows two peaks located at 159.0 and $164.3 \mathrm{eV}$ that can be assigned to $\mathrm{Bi} 4 \mathrm{f}_{5 / 2}$ and $4 \mathrm{f}_{7 / 2}$ spin-orbital components of $\mathrm{Bi}^{3+}$. The $\mathrm{Cl} 2 \mathrm{p}$ spectrum (Fig. 1d) consists of $\mathrm{Cl} 2 \mathrm{p}_{3 / 2}$ and $2 \mathrm{p}_{1 / 2}$ peaks


Fig. 2 (a and b) SEM images, (c) HRTEM image (the inset shows the SAED pattern) of BiOCl NFAs, and (d) schematic diagram of the crystal structure along the $c$ axis. located at 197.1 and $198.7 \mathrm{eV}$, respectively. The peaks agree well with previous reports. ${ }^{24,25}$

Fig. 2a shows the panoramic-view scanning electron microscopy (SEM) image of the NFAs. It reveals a high yield of hierarchical assemblies with an erythrocyte-like shape and a typical diameter of $800 \mathrm{~nm}$ to $1 \mu \mathrm{m}$. The zoomed-in SEM image (Fig. 2b) and transmission electron microscopy (TEM) image (Fig. S2, ESI $\dagger$ ) show that the assemblies consist of nanoflakes with thicknesses of $35-40 \mathrm{~nm}$. The nanoflakes are square shaped with clear corners and smooth surfaces. The hierarchical structure is still intact after a lengthy $(1 \mathrm{~h})$ ultrasonic treatment (Fig. S3, ESI + ), indicating the good structural stability. The high-resolution TEM (HRTEM) image of a single nanoflake (Fig. 2c) displays two sets of continuous lattice fringes with spacings of 0.2 and $0.28 \mathrm{~nm}$ that correspond to the (200) and (110) planes of tetragonal BiOCl, respectively. The selected-area electron diffraction (SAED) pattern (Fig. 2c, inset) reveals that the electron beam can be indexed to the [001] zone, suggesting the exposed surface to be the (001) plane. The HRTEM image at the corner of the nanoflake (Fig. S4, ESI $\dagger$ ) shows that the four lateral surfaces can be assigned to $\{110\}$ facets. A schematic diagram (Fig. 2d) provides a direct visualization of the atomic arrangement characterized in HRTEM. One can see that the minimum unit in the (001) plane is in a square shape (white lines). This microscopic feature at the atomic level agrees with the macroscopic morphology of the square-shaped nanoflakes (Fig. 2b).

The potassium storage mechanism of BiOCl NFAs is studied by using half cells at a current density of $50 \mathrm{~mA} \mathrm{~g}^{-1}$ in a voltage window of 0.01-2.0 V. The cyclic voltammetry (CV) curves of Cycle 1 (Fig. 3a) show four cathodic peaks. The strong peak at $1.5 \mathrm{~V}$ is attributed to the conversion of BiOCl to $\mathrm{Bi}^{16,17}$ The two small peaks $(0.72$ and $0.54 \mathrm{~V})$ and another strong peak at $0.1 \mathrm{~V}$ correspond to the multi-phase $\mathrm{K}-\mathrm{Bi}$ alloying reactions, while the two anodic peaks at 0.65 and $1.25 \mathrm{~V}$ correspond to the de-alloying reactions. ${ }^{26,27}$ In Cycle 2 and Cycle 3, the cathodic peak at $1.5 \mathrm{~V}$ disappears, indicating the irreversibility of the conversion reaction in Cycle 1, as will be discussed later. Other peaks remain almost unchanged except for a slight shift of the anodic peak $(0.71 \mathrm{~V})$ and the appearance of two shoulder peaks $(0.2$ and $0.83 \mathrm{~V})$. Thus, two redox pairs $0.1(0.2) / 0.71(0.83) \mathrm{V}$ and $0.72 / 1.25 \mathrm{~V}$ are established, indicating the stabilization of the alloying/de-alloying reactions. The Cycle 1 charge/discharge profiles (Fig. $3 \mathrm{~b}$ ) agree well with the observations in the $\mathrm{CV}$ curves. There are two discharge plateaus at 1.6 and $0.2 \mathrm{~V}$ as well as a slope in between, while two charge plateaus at $0.6 \mathrm{~V}$ and $1.2 \mathrm{~V}$ can be observed. The cell delivers initial discharge and charge capacities of 781 and $340 \mathrm{~mA} \mathrm{~h} \mathrm{~g}{ }^{-1}$, respectively, resulting in an initial coulombic efficiency (CE) of $43.5 \%$. Six discharge-charge states (as indicated in Fig. $3 \mathrm{~b}$ ) are selected for the ex situ XRD measurement (Fig. 3c). When discharging to $1.1 \mathrm{~V}$, the strong Bi peak demonstrates the occurrence of a conversion reaction $(\mathrm{BiOCl} \rightarrow \mathrm{Bi})$ at the initial stage of potassiation, which is correlated with the cathodic peak at $1.5 \mathrm{~V}$ in $\mathrm{CV}$ curves and the plateau at $1.6 \mathrm{~V}$ in the discharge profile. Surprisingly, not only is Bi formed but $\mathrm{K}-\mathrm{Bi}$ alloys also appear at this stage in the compositions of $\mathrm{KBi}$ and $\mathrm{K}_{3} \mathrm{Bi}_{2} \cdot{ }^{26,28}$ Early formation of alkali-metal-Bi alloys has not been 

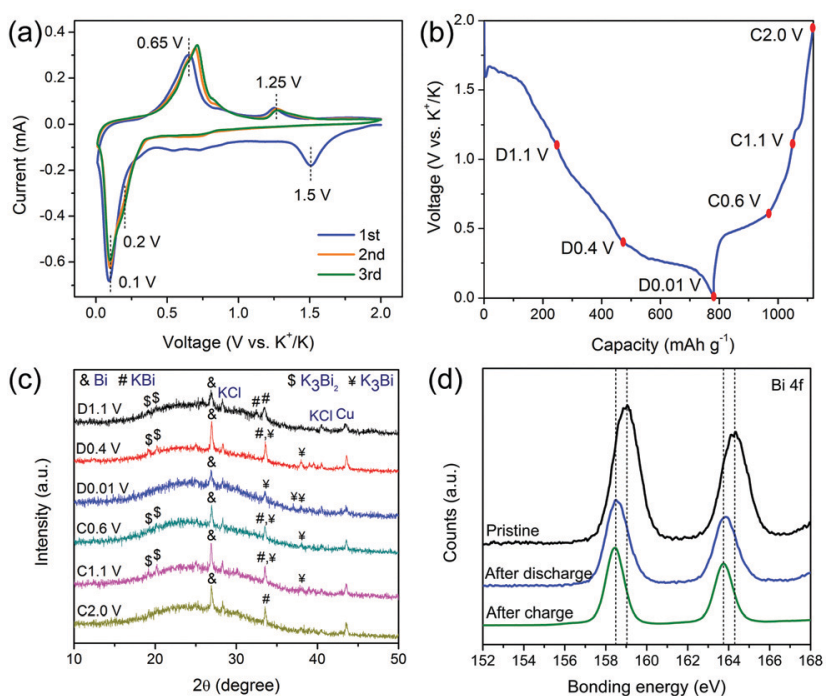

Fig. 3 (a) CV curves of Cycles 1-3, (b) discharge/charge profiles of Cycle 1, (c) XRD patterns at discharge/charge states, and (d) Bi 4f XPS spectra at the pristine state, after discharge and charge states.

observed in the lithiation and sodiation of BiOCl. ${ }^{16-18}$ Thus, it is likely that reduction of BiOCl and alloying between $\mathrm{K}$ and $\mathrm{Bi}$ occur concurrently, producing $\mathrm{Bi}$ and $\mathrm{K}-\mathrm{Bi}$ alloys. When discharging to $0.4 \mathrm{~V}$, the amount of $\mathrm{KBi}$ and $\mathrm{K}_{3} \mathrm{Bi}_{2}$ decreases while another alloy $\mathrm{K}_{3} \mathrm{Bi}$ appears. ${ }^{26,29}$ At the end of discharge $(0.01 \mathrm{~V})$, only $\mathrm{Bi}$ and $\mathrm{K}_{3} \mathrm{Bi}$ can be observed. During the charge process, de-alloying reactions occur. It leads to a gradually decreased amount of $\mathrm{K}_{3} \mathrm{Bi}$ and $\mathrm{K}_{3} \mathrm{Bi}_{2}$ and the final composition of $\mathrm{KBi}$ and $\mathrm{Bi}$ at 2.0 $\mathrm{V}$. Thus, the $\mathrm{BiOCl}$ structure is not restored, which explains the relatively low initial $\mathrm{CE}$ and the disappearance of the peak at $1.5 \mathrm{~V}$ in the $\mathrm{CV}$ curves (Fig. 3a).

Ex situ XPS results can provide additional support to the electrochemical mechanism. In Fig. $3 d$, both $\mathrm{Bi} 4 \mathrm{f}$ peaks shift to lower bonding energies after discharge, suggesting the reduction of BiOCl to Bi upon inserting K-ions. ${ }^{17}$ The peak positions remain unchanged after charging, indicating that the BiOCl structure is not restored when K-ions are extracted. The decomposition of BiOCl can also be evidenced by the evolution of the $\mathrm{O} 1 \mathrm{~s}$ peaks (Fig. S5, ESI $\dagger$ ). The major component of the $\mathrm{O}$ bonding changes from $\mathrm{Bi}-\mathrm{O}$ at the pristine state to $\mathrm{K}-\mathrm{O}$ after cycling, implying that the decomposition process is accompanied by the formation of potassium oxides. ${ }^{17,30}$ The absence of their XRD peaks may be due to their highly amorphous nature. In addition, the $\mathrm{Cl} 2 \mathrm{p}$ peak intensity (Fig. S6, ESI $\dagger$ ) dramatically decreases after discharge and charge, suggesting the formation of soluble $\mathrm{KCl}$ accompanied by the decomposition of BiOCl, which can be seen from the $\mathrm{KCl}$ peak in all the ex situ patterns (Fig. 3c). Based on the above analysis, the overall reaction pathways are schematically summarized in Fig. S7, ESI. $\dagger$

The electrochemical performance of BiOCl NFAs is fully demonstrated in Fig. 4. The discharge/charge profiles of Cycle 2 and Cycle 3 at a current density of $50 \mathrm{~mA} \mathrm{~g}^{-1}$ (Fig. 4a) well overlap with each other. A pair of plateaus at $0.2 \mathrm{~V}$ (discharge) $/ 0.6 \mathrm{~V}$ (charge) can be clearly identified. Compared to Cycle 1 (Fig. 3b), the discharge plateau at $1.6 \mathrm{~V}$ disappears, which can be ascribed


Fig. 4 (a) Discharge/charge profiles of Cycles 2 and 3, (b) cycling performance at $50 \mathrm{~mA} \mathrm{~g}^{-1}$, (c) rate performance, and (d) discharge/charge profiles at various current densities from $50 \mathrm{~mA} \mathrm{~g}^{-1}$ to $1 \mathrm{~A} \mathrm{~g}^{-1}$.

to the irreversible conversion from BiOCl to $\mathrm{Bi}$. The profiles of Cycles 2 and 3 exhibit the mixed features of those of the reported $\mathrm{Bi}$ anodes in KIBs, supporting the previously discussed electrochemical mechanism. During discharge, the slope between 1.5 and $0.4 \mathrm{~V}$ could be mainly ascribed to the formation of $\mathrm{KBi}$ and $\mathrm{K}_{3} \mathrm{Bi}_{2},{ }^{26,28}$ and the subsequent plateau is characteristic of the formation of $\mathrm{K}_{3} \mathrm{Bi}^{26,28,29}$ The charge profile agrees with those of the $\mathrm{Bi}$ anodes, suggesting a reversible dealloying process. It is interesting to note that the intermediate phases are different from one report to another $\left(\mathrm{KBi}_{2},{ }^{26} \mathrm{~K}_{5} \mathrm{Bi}_{4},{ }^{27} \mathrm{KBi}^{28}\right.$ and $\left.\mathrm{K}_{3} \mathrm{Bi}_{2}{ }^{29}\right)$, possibly due to the different size and morphology of the $\mathrm{Bi}$ anodes; thus, it is not surprising that the NFAs exhibit mixed features of the phase transition process. BiOCl NFAs deliver most of the discharge capacity below $0.4 \mathrm{~V}$ in a voltage window up to only $2 \mathrm{~V}$, which makes BiOCl favourable in assembling full-cells compared to oxides, ${ }^{31}$ dichalcogenide KIB anodes ${ }^{32-34}$ and even carbons. ${ }^{35,36}$ The NFAs delivered a Cycle 2 capacity of $367 \mathrm{~mA} \mathrm{~h} \mathrm{~g}^{-1}$ and retained $213 \mathrm{~mA} \mathrm{~h} \mathrm{~g}^{-1}$ after 50 cycles (Fig. 4b). It is worth pointing out that the obtained cyclability is far beyond the reported limitation of 15 cycles in LIBs and NIBs. ${ }^{16-18}$ The electrode was characterized by SEM after 50 cycles. The yellow dashed squares (Fig. S8, ESI $\dagger$ ) highlight that the hierarchical structure is maintained after repetitive cycles, and the flakes can be identified, which contributes to the observed cyclability. Unlike $\mathrm{Bi}$ anodes that need the incorporation of carbon to maintain the structural integrity, ${ }^{27-29}$ the NFAs exhibit good structural stability without any carbon incorporation. It can also be seen that the diameter of the NFAs increases due to the volume expansion of the $\mathrm{K}-\mathrm{Bi}$ alloying reaction, which might be responsible for the slow decay of the capacity over cycles. Strikingly, BiOCl NFAs exhibit better rate performance than in LIBs and NIBs, ${ }^{16-18}$ and even some $\mathrm{Bi}$ anodes $\left(\mathrm{Bi} / \mathrm{rGO}^{27}\right.$ and $\mathrm{Bi} / \mathrm{C}$ composites $\left.{ }^{28}\right)$. They delivered capacities of $346,314,272$ and $223 \mathrm{~mA} \mathrm{~h} \mathrm{~g}{ }^{-1}$ at current densities of 50,100, 200 and $500 \mathrm{~mA} \mathrm{~g}^{-1}$, respectively (Fig. 4c). Half of the capacity $\left(175 \mathrm{~mA} \mathrm{~h} \mathrm{~g}^{-1}\right)$ was retained when 
the current density increased to $1 \mathrm{~A} \mathrm{~g}^{-1}$. Moreover, the low discharge plateau is well maintained when the current density increases by 20 times (Fig. 4d). To the best of our knowledge, this is the first demonstration of bismuth oxyhalides that can sustain at a high rate of $1 \mathrm{~A} \mathrm{~g}^{-1}$ in alkali-metal ion batteries. The obtained performance of BiOCl NFAs can be ascribed to three factors. First, the interlayer space in the microstructure of BiOCl serves as a 2D K-ion diffusion pathway that could reduce the diffusion resistance. ${ }^{37}$ Second, the formation of $\mathrm{Bi}$ and the alloying reaction naturally enhance the electronic conductivity of the electrode matrix, and this has an even more significant role in determining the rate capability of the NFAs. Third, the macroscopic rigidity keeps the hierarchical structure of the NFAs intact despite the volume change during the alloying reaction. The combination of microstructural, mechanism and macrostructural merits results in the obtained electrochemical performance of BiOCl NFAs. Moreover, unlike the reported Bi anodes, ${ }^{26-29}$ the presented performance is obtained without carbon incorporation and ether-based electrolytes, thereby holding the advantages of the ease of synthesis and low-cost.

In summary, we have shown the promise of BiOCl as a potential KIB anode material by studying its electrochemical mechanism to store K-ions and evaluating its electrochemical performance. The mechanism study revealed that potassiation of BiOCl proceeds through a conversion reaction of transforming $\mathrm{BiOCl}$ to $\mathrm{Bi}$ and the alloying reactions of forming $\mathrm{KBi}, \mathrm{K}_{3} \mathrm{Bi}_{2}$ and $\mathrm{K}_{3} \mathrm{Bi}$. We observed that $\mathrm{K}-\mathrm{Bi}$ alloying processes proceed concurrently with the conversion process at the initial stage of potassiation, which is different from the previously reported lithiation and sodiation of bismuth oxyhalides. The electrochemical evaluation showed great cyclability and rate capability of BiOCl, exhibiting the best performance among all the reported bismuth oxyhalides in the battery research. This is the first demonstration of bismuth oxyhalides used as KIB electrode materials, and the demonstration is expected to promote future work of these compounds in this exciting field.

This work was supported by the German Research Foundation (DFG: LE2249/4-1 and LE2249/5-1) and the National Natural Science Foundation of China (21577086).

\section{Conflicts of interest}

There are no conflicts to declare.

\section{References}

1 H. Kim, J. C. Kim, M. Bianchini, D. H. Seo, J. Rodriguez-Garcia and G. Ceder, Adv. Energy Mater., 2018, 8, 1702384.

2 M. Okoshi, Y. Yamada, S. Komaba, A. Yamada and H. Nakai, J. Electrochem. Soc., 2017, 164, A54-A60.
3 M. Zhou, Y. Xu, C. Wang, Q. Li, J. Xiang, L. Liang, H. Zhao, M. Wu and Y. Lei, Nano Energy, 2017, 31, 514-524.

4 J. C. Pramudita, D. Sehrawat, D. Goonetilleke and N. Sharma, Adv. Energy Mater., 2017, 7, 1602911.

5 S. Komaba, T. Hasegawa, M. Dahbi and K. Kubota, Electrochem. Commun., 2015, 60, 172-175.

6 X. Wu, D. P. Leonard and X. Ji, Chem. Mater., 2017, 29, 5031-5042.

7 C. Zhang, Y. Xu, M. Zhou, L. Liang, H. Dong, M. Wu, Y. Yang and Y. Lei, Adv. Funct. Mater., 2017, 27, 1604307.

8 Y. Xu, C. Zhang, M. Zhou, Q. Fu, C. Zhao, M. Wu and Y. Lei, Nat. Commun., 2018, 9, 1720.

9 D. S. Bhachu, S. J. Moniz, S. Sathasivam, D. O. Scanlon, A. Walsh, S. M. Bawaked, M. Mokhtar, A. Y. Obaid, I. P. Parkin, J. Tang and C. J. Carmalt, Chem. Sci., 2016, 7, 4832-4841.

$10 \mathrm{~J}$. Li, Y. Yu and L. Zhang, Nanoscale, 2014, 6, 8473-8488.

11 R. Yuan, S. Fan, H. Zhou, Z. Ding, S. Lin, Z. Li, Z. Zhang, C. Xu, L. Wu and X. Wang, Angew. Chem., Int. Ed., 2013, 52, 1035-1039.

12 J. Zhang, F. Shi, J. Lin, D. Chen, J. Gao, Z. Huang, X. Ding and C. Tang, Chem. Mater., 2008, 20, 2937-2941.

13 M. Guan, C. Xiao, J. Zhang, S. Fan, R. An, Q. Cheng, J. Xie, M. Zhou, B. Ye and Y. Xie, J. Am. Chem. Soc., 2013, 135, 10411-10417.

14 L. Zhang, W. Wang, S. Sun, Y. Sun, E. Gao and J. Xu, Appl. Catal., B, 2013, 132, 315-320.

15 Y. Mi, L. Wen, Z. Wang, D. Cao, Y. Fang and Y. Lei, Appl. Catal., B, 2015, 176-177, 331-337.

16 L. Ye, L. Wang, H. Xie, Y. Su, X. Jin and C. Zhang, Energy Technol., 2015, 3, 1115-1120.

17 C. Chen, P. Hu, X. Hu, Y. Mei and Y. Huang, Chem. Commun., 2015, 51, 2798-2801.

18 Y. Zhang, S. Lu, M.-Q. Wang, Y. Niu, S. Liu, Y. Li, X. Wu, S.-J. Bao and M. Xu, Mater. Lett., 2016, 178, 44-47.

19 K. Zhang, J. Liang, S. Wang, J. Liu, K. Ren, X. Zheng, H. Luo, Y. Peng, X. Zou and X. Bo, Cryst. Growth Des., 2012, 12, 793-803.

20 M. Li, J. Zhang, H. Gao, F. Li, S.-E. Lindquist, N. Wu and R. Wang, ACS Appl. Mater. Interfaces, 2016, 8, 6662-6668.

21 W. Chen, W. Cai, Y. Lei and L. Zhang, Mater. Lett., 2001, 50, 53-56.

22 S. Wang, M. Wang, Y. Lei and L. Zhang, J. Mater. Sci. Lett., 1999, 18, 2009-2012.

23 J. Yuan, J. Wang, Y. She, J. Hu, P. Tao, F. Lv, Z. Lu and Y. Gu, J. Power Sources, 2014, 263, 37-45.

24 G. Cheng, J. Xiong and F. J. Stadler, New J. Chem., 2013, 37, 3207-3213. 25 S. Peng, L. Li, P. Zhu, Y. Wu, M. Srinivasan, S. G. Mhaisalkar, S. Ramakrishna and Q. Yan, Chem. - Asian J., 2013, 8, 258-268.

26 K. Lei, C. Wang, L. Liu, Y. Luo, C. Mu, F. Li and J. Chen, Angew. Chem., Int. Ed., 2018, 57, 4687-4691.

27 Q. Zhang, J. Mao, W. Pang, T. Zheng, V. Sencadas, Y. Chen, Y. Liu and Z. Guo, Adv. Energy Mater., 2018, 8, 1703288.

28 R. Zhang, J. Bao, Y. Wang and C.-F. Sun, Chem. Sci., 2018, 9, 6193-6198.

29 J. Huang, X. Lin, H. Tan and B. Zhang, Adv. Energy Mater., 2018, 8, 1703496.

30 X. Yide, Y. Li and G. Xiexian, Appl. Catal., A, 1997, 164, 47-57.

31 I. Sultana, M. M. Rahman, S. Mateti, V. G. Ahmadabadi, A. M. Glushenkov and Y. Chen, Nanoscale, 2017, 9, 3646-3654.

32 Y. Xu, F. Bahmani, M. Zhou, Y. Li, C. Zhang, F. Liang, S. H. Kazemi, U. Kaiser, G. Meng and Y. Lei, Nanoscale Horiz., 2019, 4, 202-207.

33 V. Lakshmi, Y. Chen, A. A. Mikhaylov, A. G. Medvedev, I. Sultana, M. M. Rahman, O. Lev, P. V. Prikhodchenko and A. M. Glushenkov, Chem. Commun., 2017, 53, 8272-8275.

34 J. Zhou, L. Wang, M. Yang, J. Wu, F. Chen, W. Huang, N. Han, H. Ye, F. Zhao and Y. Li, Adv. Mater., 2017, 29, 1702061.

35 Z. Jian, W. Luo and X. Ji, J. Am. Chem. Soc., 2015, 137, 11566-11569. 36 J. Zhao, X. Zou, Y. Zhu, Y. Xu and C. Wang, Adv. Funct. Mater., 2016, 26, 8103-8110.

37 H. Zhu, C. Xiao, H. Cheng, F. Grote, X. Zhang, T. Yao, Z. Li, C. Wang, S. Wei, Y. Lei and Y. Xie, Nat. Commun., 2014, 5, 3960. 